\title{
Analyse d'un disque en compression diamétrale
}

\author{
JÉrôme Chamberta ${ }^{a}$ Hichem Nouira et Jacques Duffaud \\ Université de Franche-Comté Institut FEMTO-ST - UMR 6174, Département de Mécanique Appliquée (LMARC), \\ 24 chemin de l'Épitaphe, 25000 Besançon, France
}

Reçu le 28 août 2008, accepté le 9 avril 2009

\begin{abstract}
Résumé - Dans le cadre d'une formation en mécanique, les travaux pratiques de mécanique des milieux déformables sont nécessaires aux étudiants afin qu'ils puissent mettre en pratique des notions abstraites tels que les tenseurs des contraintes et des déformations. Afin de mieux adapter ces séances de travaux pratiques aux attentes des étudiants actuels, nous avons choisi de décomposer ces séances en parties expérimentales et numériques. L'objectif de cet article est de présenter un exemple de travaux pratiques (compression d'un disque le long d'un diamètre) et de montrer les apports pédagogiques de la démarche adoptée pour l'étudiant.
\end{abstract}

Mots clés : Élasticité linéaire / extensométrie / photoélasticimétrie / méthode des éléments-finis

\begin{abstract}
Compression of a disk along a diameter. In mechanical engineering background, the lab works of continuum mechanics are necessary for the students to put abstract knowledge such as stress and strain tensors into practice. In order to adapt these lab works to the demands of students, we have chosen to decompose theses lab works into experimental and numerical parts. The main purpose of this paper is to present an example of lab works (compression of a disk along a diameter) and to show the educational benefits of the chosen approach for student.
\end{abstract}

Key words: Linear elasticity / strain measurement / photoelasticity / finite-element method

\section{Introduction}

La formation en mécanique au sein de l'université de Franche-Comté à Besançon est dispensée aux étudiants de diverses filières : IUT GMP, licence et master de Mécanique et Ingénieries, Institut Supérieur d'Ingénieurs de Franche-Comté (ISIFC, école d'ingénieurs spécialisée en instrumentation et techniques biomédicales). La mécanique des milieux déformables est souvent considérée par les étudiants comme une matière théorique et difficile. Afin de s'approprier les notions abstraites de contrainte et de déformation, il est nécessaire que l'étudiant effectue des séances de travaux pratiques. Cependant, faisant suite à un certain désintérêt des étudiants pour les travaux pratiques essentiellement expérimentaux d'élasticité linéaire, l'équipe enseignante a entrepris depuis 2003 une refonte de ces enseignements fondée sur une démarche alliant les aspects expérimentaux, théoriques et numériques.

Le but de cet article est de présenter un exemple de travaux pratiques (TP) d'élasticité linéaire d'une durée

\footnotetext{
a Auteur pour correspondance :

jerome.chambert@univ-fcomte.fr
}

de quatre heures ayant fait l'objet d'une réactualisation : un disque en alliage d'aluminium est soumis à un effort de compression appliqué par deux poinçons diamétralement opposés. Cet article s'articule autour d'une méthodologie similaire à celle proposée par $[1,2]$ en mettant l'accent sur les apports pédagogiques pour l'étudiant. Le TP commence par une pré-étude expérimentale par photoélasticimétrie d'un disque en plexiglas soumis à deux forces diamétralement opposées en utilisant la pince photoélastique Deltalab [3]. La suite du TP consiste à la mise en œuvre expérimentale par jauges de déformations sur le disque en alliage d'aluminium. Puis, nous présentons la solution théorique du disque en compression diamétrale, qui est un problème classique d'élasticité linéaire en état de contraintes planes [4]. Après avoir modélisé le disque, nous effectuons ensuite des simulations par éléments-finis en utilisant le logiciel RDM-Le-Mans (RDM6) développé par [5]. Puis, une analyse comparative entre les résultats théoriques (élasticité linéaire) et numériques (RDM6) est effectuée en termes de cartes iso-contraintes et de courbes d'évolution des contraintes. Enfin, les résultats théoriques et numériques sont comparés aux résultats expérimentaux. 


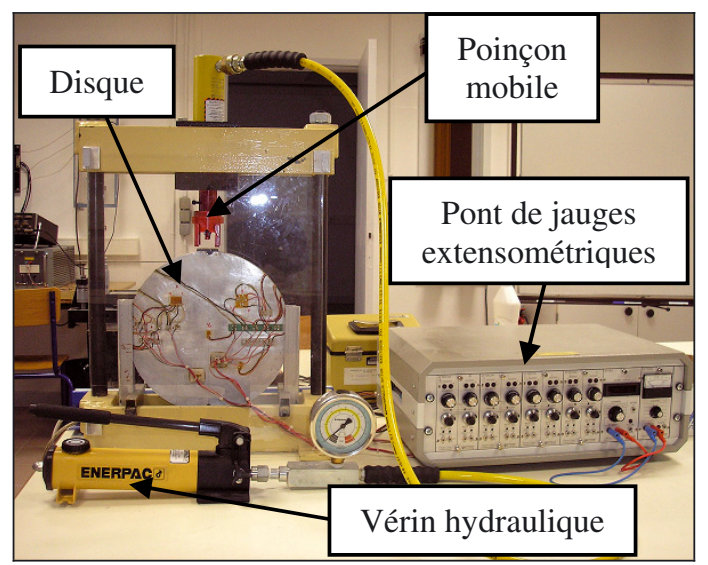

(a)

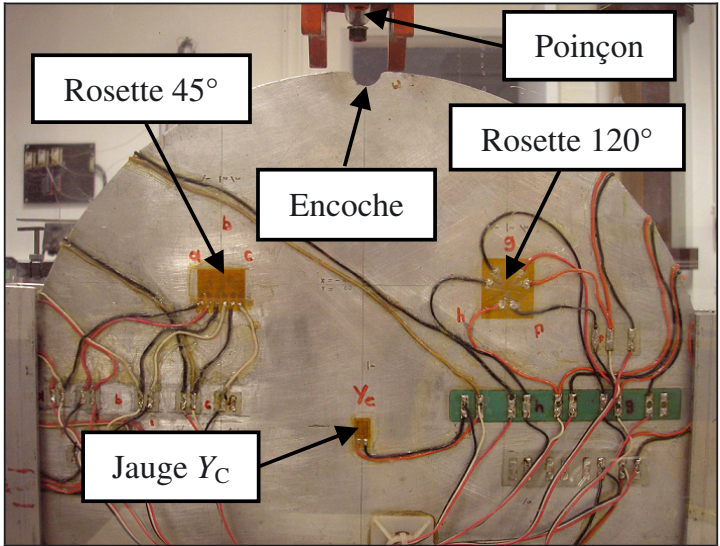

(b)

Fig. 1. Disque en AU4G soumis à des forces diamétralement opposées. (a) Dispositif expérimental. (b) Zoom sur le disque en compression.

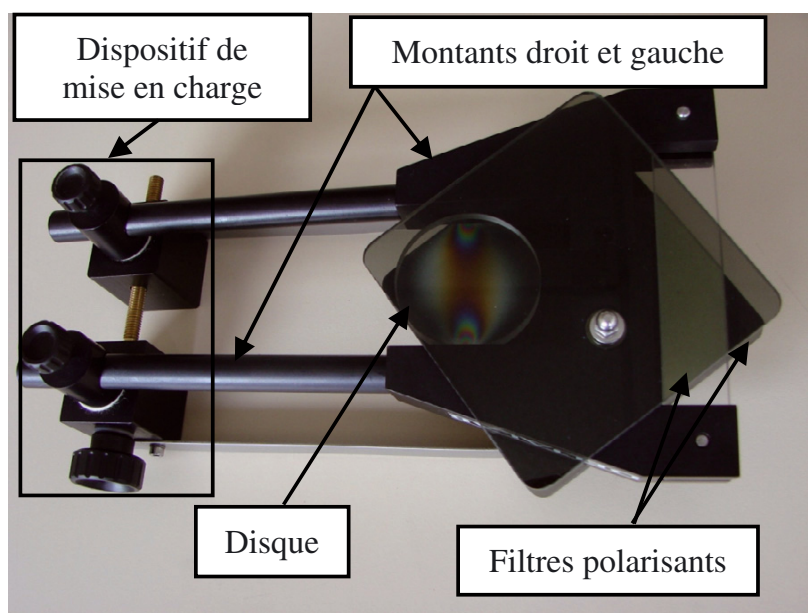

(a)

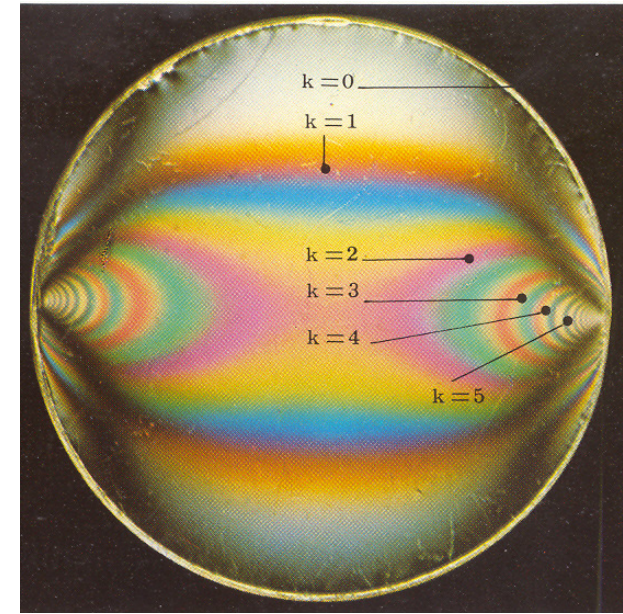

(b)

Fig. 2. Disque en compression diamétrale étudié par la pince photoélastique Deltalab. (a) Pince photoélastique. (b) Disque en compression [3].

\section{Présentation des dispositifs expérimentaux}

La figure 1 présente le principal dispositif expérimental constitué :

- d'un disque en alliage d'aluminium AU4G de rayon $R=150 \mathrm{~mm}$, d'épaisseur $e=20 \mathrm{~mm}$, de module de Young $E=74000 \mathrm{MPa}$, de coefficient de Poisson $\nu=$ 0,33 et de limite d'élasticité en traction $\sigma_{\mathrm{e}}=240 \mathrm{MPa}$. Le disque possède deux encoches diamétralement opposées de rayon $a=5 \mathrm{~mm}$;

- d'un système de mise en charge composé d'un bâti et d'un vérin hydraulique actionnable manuellement pour l'application du chargement de compression diamétrale par l'intermédiaire de deux poinçons demicylindriques (un mobile et un fixe);
- d'un pont de jauges permettant de mesurer les déformations au niveau d'une jauge simple collée au centre du disque suivant la direction verticale (jauge $\left.Y_{\mathrm{C}}\right)$; et d'une rosette à $120^{\circ}$, composée de trois jauges $(f, g, h)$, collée au point de coordonnées $\left(x_{1}=60 \mathrm{~mm}\right.$, $\left.x_{2}=60 \mathrm{~mm}\right)$ avec la jauge $g$ dirigée suivant la direction verticale.

La pince photoélastique Deltalab [3], présentée sur la figure 2a, est composée :

- d'un disque en plexiglas de diamètre $\phi=45 \mathrm{~mm}$, d'épaisseur $e=5,5 \mathrm{~mm}$;

- de deux filtres polarisants appelés « polariseur» et « analyseur»;

- de deux montants qui permettent de bloquer le disque; 


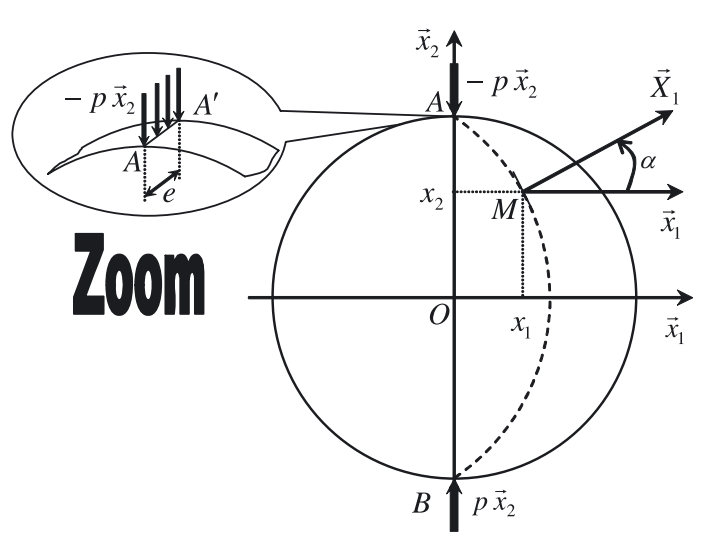

Fig. 3. Géométrie.

- d'un dispositif de mise en charge constitué d'un bouton et vis de serrage, d'une vis d'immobilisation des poignées de la pince.

\section{3 Étude analytique}

Sur la figure 3, considérons un disque de rayon $R$ soumis à deux forces $-p \vec{x}_{2}$ et $p \vec{x}_{2}$ agissant respectivement en deux points $A$ et $B$ diamétralement opposés. Sachant que le module $p$ désigne une force par unité d'épaisseur, l'effort global de compression appliqué sur le disque est égal à $F=p e$. On suppose que le disque est en état de contraintes planes. Le champ de contraintes solution de ce problème d'élasticité plane est obtenu en superposant les trois états de contraintes suivants :

- une distribution radiale simple produite par la force $-p \vec{x}_{2}$ appliquée au point $A$;

- une distribution radiale simple produite par la force $p \vec{x}_{2}$ appliquée au point $B$;

- une traction radiale uniforme $p /(\pi R)$ sur la frontière du disque.

Dans la base orthonormée directe $\left(\vec{x}_{1}, \vec{x}_{2}, \vec{x}_{3}\right)$, les composantes non nulles du tenseur des contraintes s'écrivent finalement, en tout point $M\left(x_{1}, x_{2}\right)$ du disque, sous la forme suivante :

$$
\begin{aligned}
& \sigma_{11}=\frac{p}{\pi R}-\frac{2 p}{\pi} \frac{\left(R-x_{2}\right) x_{1}^{2}}{\left[x_{1}^{2}+\left(R-x_{2}\right)^{2}\right]^{2}}-\frac{2 p}{\pi} \frac{\left(R+x_{2}\right) x_{1}^{2}}{\left[x_{1}^{2}+\left(R+x_{2}\right)^{2}\right]^{2}} \\
& \sigma_{22}=\frac{p}{\pi R}-\frac{2 p}{\pi} \frac{\left(R-x_{2}\right)^{3}}{\left[x_{1}^{2}+\left(R-x_{2}\right)^{2}\right]^{2}}-\frac{2 p}{\pi} \frac{\left(R+x_{2}\right)^{3}}{\left[x_{1}^{2}+\left(R+x_{2}\right)^{2}\right]^{2}} \\
& \tan \alpha=\frac{2 x_{1} x_{2}}{x_{1}^{2}-x_{2}^{2}+R^{2}} \\
& \sigma_{12}=\frac{2 p}{\pi} \frac{\left(R-x_{2}\right)^{2} x_{1}}{\left[x_{1}^{2}+\left(R-x_{2}\right)^{2}\right]^{2}}-\frac{2 p}{\pi} \frac{\left(R+x_{2}\right)^{2} x_{1}}{\left[x_{1}^{2}+\left(R+x_{2}\right)^{2}\right]^{2}}
\end{aligned}
$$

où $\alpha=\left(\vec{x}_{1}, \vec{X}_{1}\right)$ est l'angle entre la direction $\vec{x}_{1}$ et la direction principale $\vec{X}_{1}$ (voir Fig. 3). Il est important de remarquer que le modèle proposé avec deux forces concentrées $\pm p \vec{x}_{2}$ (cas 1 sur Fig. 4) est une idéalisation

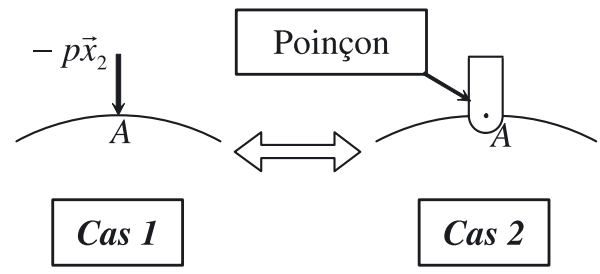

Fig. 4. Application du chargement.

de la réalité. Sur le montage expérimental de la figure 1, le disque est soumis à des forces réparties appliquées par les poinçons (cas 2 sur Fig. 4) qui se logent dans des encoches de forme complémentaire usinées dans le disque (voir Fig. 1b).

Afin de préparer cette séance de travaux pratiques sur la compression d'un disque le long d'un diamètre, la résolution de ce problème d'élasticité plane peut être préalablement proposée aux étudiants en travaux dirigés.

\section{Démarche expérimentale}

Dans un premier temps, les étudiants commencent le TP par une initiation à la photoélasticité. Ils utilisent la pince photoélastique Deltalab [3] afin de visualiser les isochromatiques d'un disque en plexiglas soumis à deux forces diamétralement opposées. Les isochromatiques sont des franges d'interférences qui correspondent à l'extinction de certaines longueurs d'onde de la lumière blanche et qui se produisent lorsque :

$$
\sigma_{1}-\sigma_{2}=\frac{k \lambda}{e C}
$$

où $\sigma_{1}$ et $\sigma_{2}$ sont les contraintes principales, $k$ un entier représentant l'ordre d'une isochromatique, $\lambda$ la longueur d'onde dont on observe l'extinction, e l'épaisseur du disque et $C$ une constante photoélastique caractéristique du matériau. La photoélasticité permet donc de visualiser la différence des contraintes principales, qui représente également la contrainte équivalente de Tresca $\left(\sigma_{\text {Tresca }}=\right.$ $\left.\left|\sigma_{1}-\sigma_{2}\right|\right)$. La figure $2 \mathrm{~b}$ illustre un exemple d'isochromatiques (de différents ordres $k=0,1,2,3$, etc.) du disque chargé en compression.

Dans un second temps, les étudiants font des manipulations sur le dispositif expérimental avec le disque en AU4G. Après avoir réglé manuellement le pont de jauges, ils appliquent une pression de 300 bars, qui est mesurée à l'aide d'un manomètre. Les valeurs expérimentales des déformations données par les jauges $Y_{\mathrm{C}}, f, g$ et $h$ sont listées dans le tableau 1.

En utilisant le logiciel RDM6 - Dépouillement des rosettes [5] avec les mesures expérimentales $\varepsilon_{\mathrm{f}}$, $\varepsilon_{\mathrm{g}}$, et $\varepsilon_{\mathrm{h}}$, on obtient les composantes expérimentales, au point $(60,60)$, du tenseur des déformations dont les valeurs sont listées dans le tableau 1. Le dispositif expérimental n'est pas équipé de capteur de force pour mesurer l'effort appliqué sur le disque. Afin de déterminer cet effort, il est proposé aux étudiants de comparer les valeurs expérimentale 
Tableau 1. Valeurs expérimentales des déformations (exprimées en $10^{-6}$ ).

\begin{tabular}{llllllll}
\hline$\varepsilon_{\mathrm{Yc}}(0,0)$ & $\varepsilon_{\mathrm{f}}(60,60)$ & $\varepsilon_{\mathrm{g}}(60,60)$ & $\varepsilon_{\mathrm{h}}(60,60)$ & $\varepsilon_{11}(60,60)$ & $\varepsilon_{22}(60,60)$ & $\varepsilon_{33}(60,60)$ & $\varepsilon_{12}(60,60)$ \\
\hline$-229,82$ & $-46,79$ & $-142,94$ & 64,56 & 59,49 & $-142,94$ & 41,1 & 64,29 \\
\hline
\end{tabular}

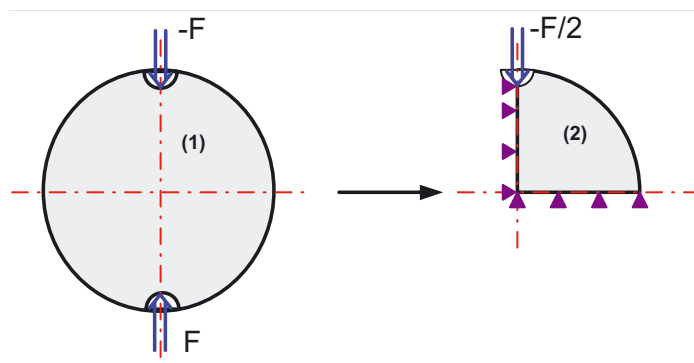

Fig. 5. Modélisation.

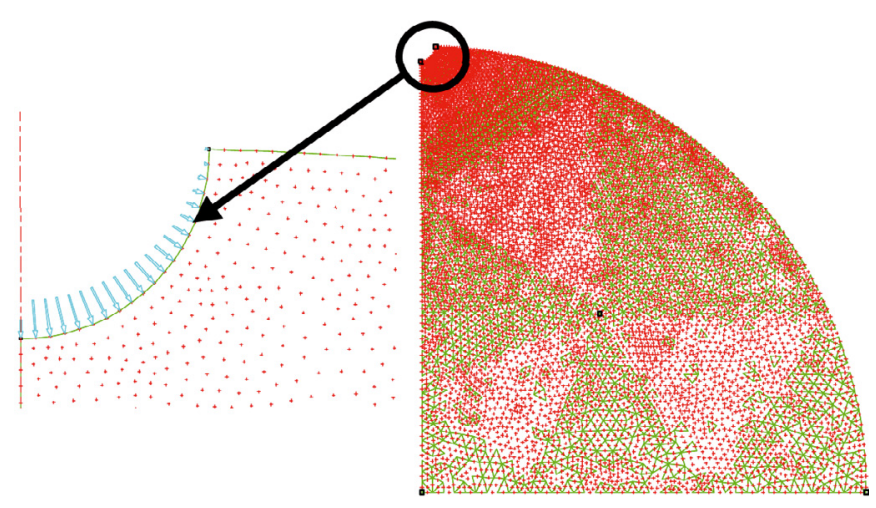

Fig. 6. Chargement et maillage.

et analytique de la déformation $\varepsilon_{22}$ au centre du disque. La valeur expérimentale correspond à la déformation $\varepsilon_{Y c}$ donnée par la jauge $Y_{\mathrm{C}}$. L'expression analytique $\varepsilon_{22}(0,0)$ est obtenue en utilisant la loi de Hooke généralisée et l'équation (1). Finalement, on obtient :

$$
\begin{aligned}
& p=-\frac{\pi R E \varepsilon_{Y_{\mathrm{C}}}}{3+\nu}=2406,67 \mathrm{~N} \cdot \mathrm{mm}^{-1} ; \\
& F=p e=48133 \mathrm{~N}
\end{aligned}
$$

Ces résultats seront utilisés pour les calculs analytiques (théorie) et numériques (RDM6).

\section{Modélisation numérique}

Du fait des symétries de géométrie et de chargement, le système étudié est modélisé par un quart de disque (voir Fig. 5). La modélisation des conditions aux limites, schématisées sur la figure 5, constitue une des principales difficultés rencontrées par les étudiants. Le maillage du quart de disque, effectué par une triangulation de Delaunay, est composé de 6578 éléments triangulaires (à 6 nœuds) et de 13405 nœuds (voir Fig. 6). Le maillage est raffiné au niveau de l'encoche. L'hypothèse des contraintes planes est adoptée. Le chargement appliqué par le poinçon sur le disque est modélisé par un

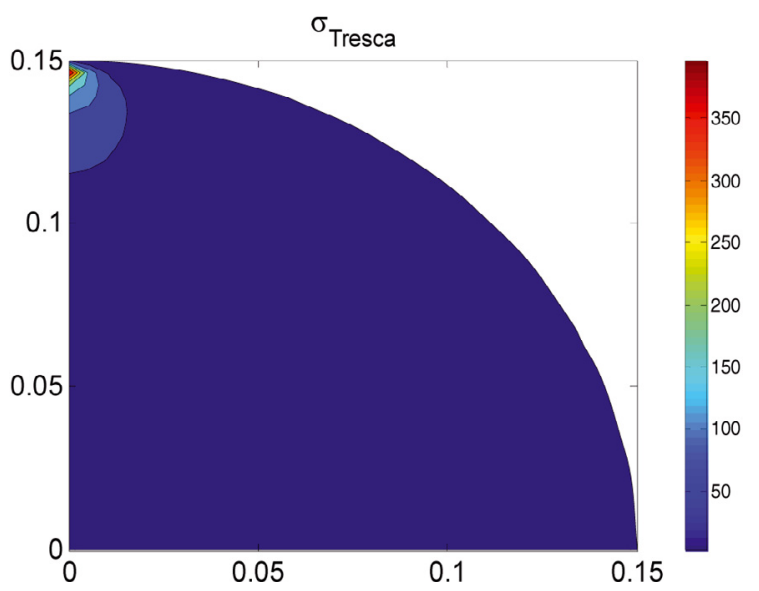

Fig. 7. Iso-contrainte $\sigma_{\text {Tresca }}$ analytique.

effort réparti le long de l'encoche (voir Fig. 6). Quel que soit le point $M(r=a, \theta)$ appartenant à la surface de l'encoche $S_{\text {en }}$, le chargement est modélisé par une distribution radiale simple dont le vecteur contrainte est défini, dans la base cylindrique $\left(\vec{e}_{\mathrm{r}}, \vec{e}_{\theta}, \vec{x}_{3}\right)$, par :

$$
\vec{T}\left(M \in S_{\mathrm{en}},-\vec{e}_{\mathrm{r}}\right)=\overline{\bar{\sigma}} \cdot\left(-\vec{e}_{\mathrm{r}}\right)=-\sigma_{\mathrm{rr}} \vec{e}_{\mathrm{r}}=\frac{2 F}{\pi a e} \cos \theta \vec{e}_{\mathrm{r}}
$$

où $\sigma_{\mathrm{rr}}$ est la contrainte normale en $M$ sur la facette de normale $\vec{e}_{\mathrm{r}}=\overrightarrow{A M} / a$ et $\theta=\left(-\vec{x}_{2}, \vec{e}_{\mathrm{r}}\right)$.

\section{Résultats et discussion}

\subsection{Confrontation entre les études analytiques et numériques}

Sur la figure 7, la distribution de la contrainte équivalente de Tresca $\sigma_{\text {Tresca }}$ obtenue à partir de l'équation (1) correspondant à la solution analytique est visualisée à l'aide du logiciel MATLAB. La figure 8 représente la distribution de la contrainte $\sigma_{\text {Tresca }}$ déterminée par RDM6. Nous constatons un bon accord entre les résultats analytiques (théorie de l'élasticité linéaire), numériques (méthode des éléments-finis RDM6) et expérimentaux (voir Fig. 2b).

Les figures 9 et 10 montrent respectivement les courbes d'évolution des contraintes $\sigma_{11}$ et $\sigma_{22}$ en fonction de l'ordonnée $x_{2}$ obtenues par la théorie de l'élasticité et par la méthode des éléments-finis (RDM6). Sur la figure 9 , on remarque que les valeurs de $\sigma_{11}$ obtenues par la théorie et par RDM6 sont presque identiques quelle que soit la valeur de $x_{2}$ comprise entre 0 et $125 \mathrm{~mm}$. Pour des valeurs proches de la zone d'application du chargement $\left(x_{2}=145 \mathrm{~mm}\right)$, les deux courbes diffèrent de 
Tableau 2. Récapitulatif des résultats au point (60, 60).

\begin{tabular}{cccccc}
\hline & Valeur expérimentale & \multicolumn{2}{c}{ Valeur analytique } & \multicolumn{2}{c}{ Valeur numérique } \\
& $M(60,60)$ & $M(60,60)$ & Écart relatif & $M(60,60)$ & Écart relatif \\
\hline$\sigma_{11}$ & $1,023 \mathrm{MPa}$ & $0,97 \mathrm{MPa}$ & $5,18 \%$ & $0,99 \mathrm{MPa}$ & $3,23 \%$ \\
$\sigma_{22}$ & $-10,24 \mathrm{MPa}$ & $-9,29 \mathrm{MPa}$ & $9,28 \%$ & $-9,28 \mathrm{MPa}$ & $9,38 \%$ \\
$\sigma_{12}$ & $3,58 \mathrm{MPa}$ & $3,66 \mathrm{MPa}$ & $2,23 \%$ & $3,63 \mathrm{MPa}$ & $1,4 \%$ \\
$\alpha$ & $16,21^{\circ}$ & $17,74^{\circ}$ & $9,44 \%$ & $17,76^{\circ}$ & $9,56 \%$ \\
\hline
\end{tabular}

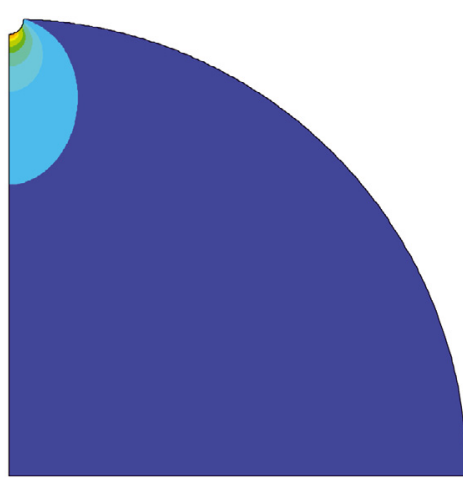

SIOHA RESECA

Fig. 8. Iso-contrainte $\sigma_{\text {Tresca }}$ numérique.

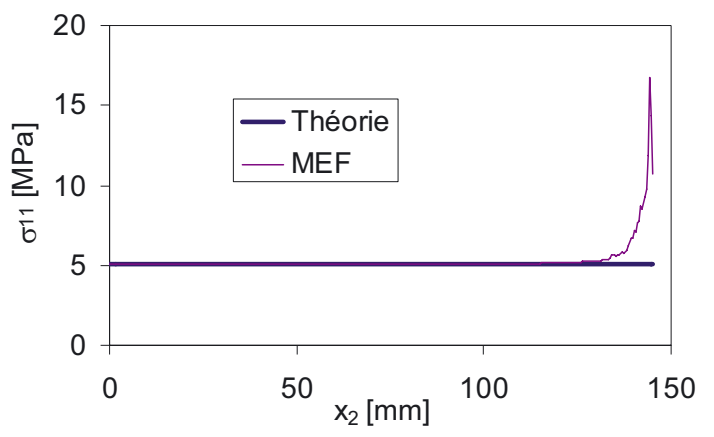

Fig. 9. Évolution de $\sigma_{11}$ selon l'axe $x_{1}=0$.

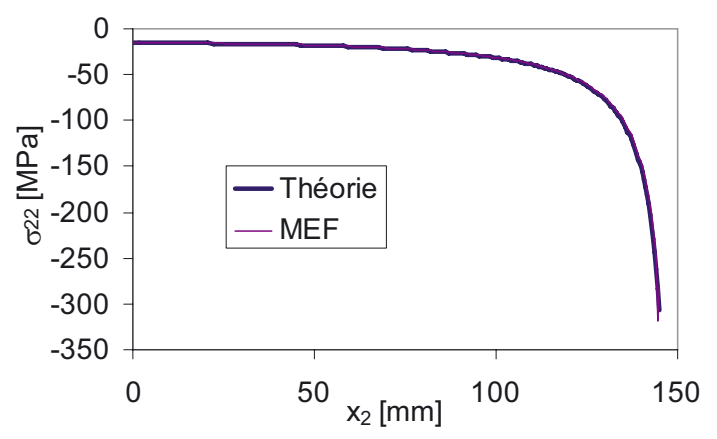

Fig. 10. Évolution de $\sigma_{22}$ selon l'axe $x_{1}=0$.

manière significative. Sur la figure 10, on constate une très bonne concordance entre les deux courbes pour la composante $\sigma_{22}$.

\subsection{Comparaison au point $(60,60)$}

Les étudiants doivent effectuer une analyse comparative entre les résultats expérimentaux, analytiques (théorie de l'élasticité) et numériques (RDM6) au point de coordonnées $(60,60)$. Les résultats obtenus sont listés dans le tableau 2. Les valeurs expérimentales des contraintes sont déduites des valeurs expérimentales des déformations (voir Tab. 1) en utilisant la loi de Hooke généralisée. L'angle $\alpha$ expérimental est obtenu grâce au logiciel RDM6-Rosettes. Les valeurs analytiques sont calculées à l'aide de l'équation (1). Les valeurs numériques sont déterminées grâce au logiciel RDM6. L'écart relatif est calculé par rapport à la valeur expérimentale. On constate que les résultats analytiques et numériques au point $(60,60)$ sont en bon accord avec les résultats expérimentaux avec des écarts relatifs toujours inférieurs à $10 \%$. En outre, on remarque que les valeurs analytiques et numériques sont quasiment identiques.

\section{Conclusion}

$\mathrm{Au}$ cours de la séance de TP relative à un disque en compression diamétrale, les étudiants travaillent en binôme et doivent mettre en œuvre des démarches expérimentales (photoélasticimétrie et jauges de déformations), analytiques (théorie de l'élasticité linéaire) et numériques (méthode des éléments-finis). À l'issue de la séance, les étudiants sont amenés à faire la synthèse des résultats obtenus dans un compte rendu. Cette séance doit permettre aux étudiants de comprendre, d'une part que l'approche expérimentale reste essentielle en mécanique, et d'autre part que toute simulation numérique dépend du degré de complexité de la modélisation utilisée.

\section{Références}

[1] L. Chevalier, B. Raka, Confrontation RdM-MMC : une démarche expérimentale de validation, Technologie 129 (2004) 33-41

[2] J. Chambert, H. Nouira, J. Duffaud, Démarche expérimentale, théorique et numérique dans le cadre de travaux pratiques en élasticité linéaire : exemple du disque en compression, Actes du $18^{\text {ème }}$ Congrès Français de Mécanique, Grenoble, France, 27-31 août 2007

[3] S. Konieczka, Pince photoélastique - Initiation à la photoélasticité, Deltalab, 1992

[4] S. Timoshenko, J.N. Goodier, Théorie de l'élasticité, Librairie Polytechnique, Ch. Béranger, Paris, 1961

[5] Y. Debard, Guide d'utilisation de RDM6 - Éléments finis, Version 6.13, 2001 